\title{
Vagal pathway could explain gut dysfunction in Parkinson disease
}

Before the onset of motor symptoms, constipation and gastric dysmotility are known to manifest in many patients with early-stage Parkinson disease (PD). A new study has now identified a monosynaptic nigrovagal pathway in rats that contributes to gastric tone and motility, and might explain gastric symptoms in patients with PD.

The association of gut symptoms with PD suggests a pathophysiological connection between the substantia nigra pars compacta $(\mathrm{SNc})$, a site of neuronal loss in $\mathrm{PD}$, and the dorsal motor nucleus of the vagus (DMV), which provides the vagal motor output to the gastrointestinal tract. Braak's hypothesis states that sporadic PD might be triggered by an unknown pathogen reaching the gut, which travels to the brain through the vagus nerve. "In order for the unknown pathogen to reach the SNc from the DMV, there has to be a neuronal pathway connecting these two areas," explains author R. Alberto Travagli.

To demonstrate the existence of a nigro-vagal pathway, the investigators used neuronal tracing experiments in rats. Microinjections of a retrograde tracer in the DMV labelled neurons in the SNc, and an anterograde tracer in the SNc labelled fibres that appose DMV neurons. As neither tracer crossed synapses, the data suggest a monosynaptic nigro-vagal pathway.

The researchers then showed that microinjections of neuroactive agents into the $\mathrm{SNc}$ of rats resulted in dose-dependent increases in tone and motility of the gastric corpus and antrum. These gastric effects were abolished or attenuated after vagotomy, or by optogenetic inhibition of nigro-vagal terminals following microinjections of halorhodopsin into the SNc. These results indicate the physiological relevance of the nigro-vagal pathway.

Finally, in rats treated with paraquat to induce experimental Parkinsonism, gastric effects mediated by neuroactive stimulation of the SNc were markedly reduced compared with untreated control rats. As gastric impairment arose before motor symptoms, this finding implies that the nigro-vagal pathway was compromised during PD development.

"The next steps in our work will focus on identifying the environmental factors that promote the degeneration of this pathway and the approaches aimed at attenuating or preventing its degeneration altogether," concludes Travagli.

\section{Iain Dickson}

ORIGINAL ARTICLE Anselmi, L. et al.

A nigro-vagal pathway controls gastric motility and is affected in a rat model of Parkinsonism. Gastroenterology http://dx.doi.org/10.1053/

j.gastro.2017.08.069 (2017)

FURTHER READING Travagli, R. A. \& Anselmi, L. Vagal neurocircuitry and its influence on gastric motility. Nat. Rev. Gastroenterol. Hepatol. 13,

389-401 (2016) 